\title{
A Hypothetical Approach to Cosmic Inflation Incorporating Binary Entropy
}

\author{
Jerome Cantor ${ }^{1}$ \\ ${ }^{1}$ St John's University, Queens, NY, USA \\ Correspondence: Jerome Cantor, St John's University, 8000 Utopia Pkwy, Queens, NY 11439, USA. E-mail: \\ jocantor1@gmail.com
}

Received: October 11, 2021

Accepted: November 10, 2021

Online Published: December 14, 2021

doi:10.5539/apr.v13n3p9

URL: https://doi.org/10.5539/apr.v13n3p9

\begin{abstract}
The current paper presents an alternative hypothesis for the termination of cosmic inflation based on Huang's model of spacetime involving the movement of a superfluid through a random resistor network. Using this model, we previously derived a mathematical relationship between the velocity of a reference frame and the probability that a random bond is intact. As an extension of this finding, the permutations of open and closed bonds are now shown to represent potential microstates, thus providing a means of relating motion within the network to binary entropy. Applying this concept to cosmic inflation, termination of this process is an expected consequence of the changes in binary entropy associated with the increasing velocity of expansion.
\end{abstract}

Keywords: cosmic inflation, binary entropy, superfluid, random resistor network, spacetime

\section{Introduction}

The idea of cosmic inflation arose from the need to reconcile the homogeneity of the universe with the limitations of the standard model of expansion (Linde, 1983; Linde, 1982; Guth, 1981; Einhorn, Stein, \& Toussaint, 1980; Starobinsky, 1979). As proposed by Guth and others, inflationary theory postulates a brief period of supercooling at the onset of expansion that produced a uniform distribution of matter, an absence of magnetic monopoles, and a flattened universe. Various models have been constructed to describe this process, but the factors responsible for its initiation and termination remain uncertain (Patel \& Lineweaver, 2017; Uzan, 2015; Easson, Frampton, \& Smoot, 2012; Tegmark, 2005; Carroll \& Chen, 2004).

One of the important issues arising from this inflation model is the source of the immense repulsive force responsible for the nearly instantaneous expansion of matter. This event transcends conventional gravitational effects and requires postulating a "false vacuum" involving unstable quantum activity. Furthermore, the rapid supercooling following inflation necessitates an unexplained transition to a "true vacuum" that abrogates the thermodynamic energy associated with inflation.

In the current paper, we present a model of spacetime that provides an alternative mechanism for cosmic inflation. It involves changes in binary entropy associated with the transition of a superfluid to a normal one which account for the initiation and termination of cosmic inflation without imposing a transition in vacuum states (Cantor, 2016).

The unique properties of superfluids, which exhibit quantum behavior at the macroscopic level, may be incorporated into a percolation model of spacetime, where the random interaction of forces at the subatomic level give rise to complex phenomena at higher levels of scale (Liberati \& Maccione, 2014; Volovik, 2013; Annett, 2004; Kapitulnik, Aharony, Deutscher, et al., 1983). At a critical point in the evolution of the percolation system, a phase transition occurs which produces new patterns of physical or chemical behavior (Papon, Leblond, \& Meijer, 2006). Consequently, these types of networks may be used to investigate emergent phenomena such as cosmic inflation.

With regard to modeling superfluid behavior to encompass inflation, Huang et al proposed that vortices within the fluid become entangled during this process, thereby creating a homogeneous environment consistent with development of an isotropic universe (Huang, Low, \& Roh-Suan, 2012). Following inflation, these vortices become separated, allowing for the condensation of matter associated with noninflationary expansion. 
One advantage of the model is the emergence of a formula for calculating the probability that a random bond is open or closed, based on the velocity of a reference frame within the network (Cantor, 2016). Using this property, it was possible to relate the process of cosmic expansion to the degree of binary entropy within the system, thereby providing a mechanism for the inflationary process.

\section{The Inflationary Process}

To accommodate the isotropic properties of the universe, the fundamental concept of cosmic expansion was modified by inserting a momentary period of exponential inflation involving the propagation and preservation of a homogeneous distribution of matter. While there is no empirical evidence for this process, the proposed mechanism could nevertheless be crafted from morphometric and thermodynamic analysis of the incipient universe.

Perhaps the greatest obstacle in establishing this theory is the need to postulate an incredibly powerful force that counteracts any gravitational effect associated with outward movement from a singularity. Among the suggested mechanisms to explain this breach of physical laws is the concept of a "false vacuum" in which a combination of mass density and negative pressure produces a large repulsive force (Bousso, Harlow, \& Senatore, 2015)

However, the false vacuum requires the insertion of a cosmological constant that is $10^{100}$ times larger than the one that is applicable to the current universe. To reconcile this disparity, it was proposed that the repulsive force causes a precipitous drop in temperature which induces a phase transition to a true vacuum state, allowing the emergence of particles and antiparticles associated with conventional noninflationary cosmologies (Spolyar, 2011).

\section{Superfluid Network Model of Spacetime}

To avoid the imposition of specialized physical conditions, it may be possible to construct an alternative model of cosmic inflation involving changes in entropy rather than gravitational force. Under these conditions, spontaneous changes in the number of microstates are responsible for propagating transient exponential growth of the universe. The substitution of entropy for gravitational effects has been previously discussed in the literature, particularly with regard to noninflationary expansion (Carroll \& Chatwin-Davies, 2018).

In our own studies, we employed a model of spacetime proposed by Huang that involves the movement of a superfluid through a 3-dimensional random resistor network composed of uniform interconnecting bonds that are indiscriminately severed to simulate a rise in fluid viscosity (Redner, 2007; Farago \& Kantor, 2000; Golden, 1997, Huang, 1995; de Gennes, 1976). The model was used to derive a mathematical relationship between the velocity of a reference frame and the probability of intact bonds, where permutations of open and closed bonds represent potential microstates (Cantor, 2016). Applying this concept to cosmic inflation, initiation and termination of this process is an expected consequence of changes in binary entropy associated with the increasing velocity of expansion.

To construct this model of superfluid behavior, an external electromotive force was substituted for flow pressure. Current density ( $j$ ) could then be expressed as:

$$
j=\rho_{s} v_{s}+\rho_{n} v_{n}
$$

where $\rho_{s} v_{s}$ and $\rho_{n} v_{n}$ represent the density $(\rho)$ and velocity $(v)$ of the superfluid and normal fluid components, respectively (Huang, 1995).

If the channels in the network are sufficiently small, $v_{n}=0$, and the normal fluid component is removed from the equation, then

$$
j=\rho_{s} \nabla \Phi
$$

corresponding to Ohm's law, where $\Phi$ represents the electrostatic potential (Huang, 1995).

As conducting bonds are cut indiscriminately, their total cross-sectional area is reduced, resulting in a decrease in superfluid density and a reciprocal increase in normal fluid density such that

$$
\rho_{s}+\rho_{n}=\text { constant }
$$




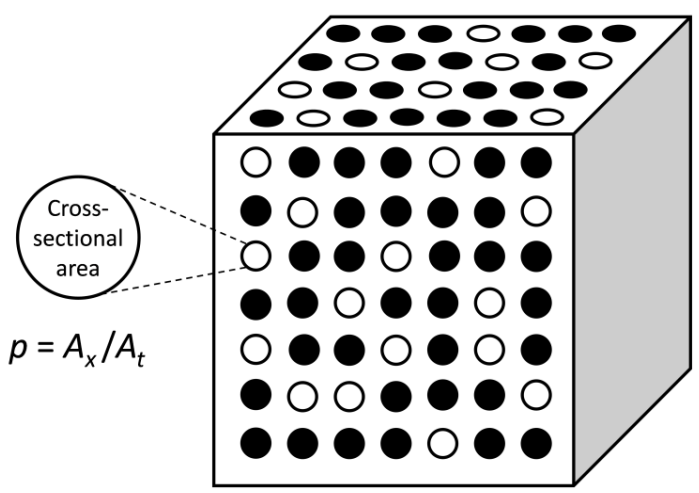

Figure 1. Diagram of the superfluid network model, showing uniform interconnecting bonds that are either intact (white) or disconnected (black). The cross-sectional area of the nonconducting bonds $\left(A_{x}\right)$, as a proportion of the total cross-sectional area $\left(A_{t}\right)$, is a measure of probability $(p)$, fluid density, and viscosity. Reprinted from

(Cantor, 2016).

Thus, fluid density and viscosity may be described in terms of the cross-sectional area of the uniform open and closed bonds (Figure 1). In this binary configuration, the relative number of open bonds is a measure of the probability that a random bond is intact $(p)$ or disconnected $(1-p)$.

This relationship was incorporated into Poiseuille's law to express fluid pressure in the system and combined with Bernoulli's principle to show that the relative number of open bonds is inversely proportional to the velocity of a given reference frame within the network (Cantor, 2016). Surprisingly, the formula for determining the probability that a random bond is open is identical to that associated with relativistic velocity transformation (equation 4), which may suggest a deeper connection between the model and spacetime.

$$
p=\sqrt{1-\frac{v^{2}}{c^{2}}}
$$

\section{Modeling Binary Entropy in Cosmic Inflation}

Since a bond may be either open or closed, it is possible to use the simple coin-flip analogy as a means of analyzing the probabilistic features of the network. The number of permutations is directly related to the motion of the system according to equation 4 , where the degree of freedom is reduced to a single entity at a velocity of either zero or $\mathrm{C}$, when all the bonds share the same microstate. The entropy in the system may be expressed in terms of microstates as follows:

$$
S=k \ln \Omega
$$

where $k$ is Boltzmann's constant and $\Omega$ is the number of microstates.

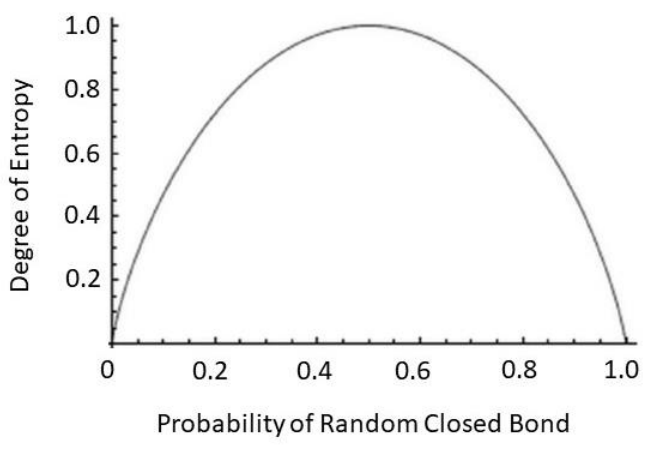

Figure 2. Graph of change in binary entropy (equation 6) with increasing probability of a random network bond being closed. Velocity may be substituted for probability on the $\mathrm{x}$-axis according to equation 4. 
As the velocity of the system increases from zero, the number of permutations rapidly expands according to the binomial distribution and the available microstates increase monotonically, where maximum entropy is reached when there are similar numbers of open and closed bonds (Figure 2). As the probability of a closed bond increases beyond 0.5 , the number of microstates begins to decline monotonically, and again reaches one at maximum frame velocity. This process involves mutually exclusive events with probabilities $p$ and 1-p and may be expressed in terms of binary entropy, $H(p)$, as follows:

$$
H(p)=-p \log _{2} p-(1-p) \log _{2}(1-p)
$$

While binary entropy is generally associated with information theory, the current model provides a physical construct for this concept involving the calculation of all possible combinations of open and closed bonds in the network. Given the fact that multiple probability states may exist simultaneously, even small increments in velocity are associated with substantial increases in entropy such that the expanding universe can move from an initial temperature of absolute zero to an immensely hot state almost instantaneously. The sudden presence of virtually infinite microstates would produce the enormous thermodynamic pressures within the system associated with inflationary models of expansion.

However, this process would be self-contained due to a decrease in the formation of new microstates when the probability of a random bond being closed exceeded 0.5 . This would rapidly reduce the entropy per unit volume, resulting in a significant drop in temperature, consistent with supercooling. While the density of microstates within the system would decrease, their total number would continue to grow with noninflationary expansion and the overall entropy of the system would increase in accordance with the second law of thermodynamics in a closed system such as the universe.

Furthermore, the decrease in the velocity of expansion following inflation would result in a more balanced distribution between open and closed network bonds (from equation 4), thus reversing the decrease in entropy density and inducing the transition to a more rapidly expanding universe that occurred approximately 4 billion years ago (Riess, Filippenko, Challis, et al., 1998; Perlmutter, Aldering, Della Valle, et al., 1998).

\section{Discussion}

Our model provides a more straightforward explanation of the transition from a post-inflationary decline in cosmic expansion to a more rapidly growing universe, based on the relationship between velocity and microstates (Figure 2). However, testing the validity of any entropy-based model of inflation may be difficult because it relies on the abstract concept of disorder which is commonly measured in terms of its indirect effects on other physical parameters.

Nevertheless, it may be possible to determine whether the current model is consistent with cosmological observations and therefore of theoretical interest. The continued increase in expansion velocity involving a decline in microstates might be validated by the use of more powerful telescopes to determine whether the density of matter at the cosmic horizon exceeds the predicted value based on current measurements. Such a discrepancy already exists in the form of the Hubble tension, which may require new theoretical approaches to cosmic expansion (Jedamzik, Pogosian, \& Zhao, 2021).

With regard to the limitations of our model, there is no mechanism to explain the movement away from a single microstate at time zero. However, an initial resting state may be hypothesized in which there are two separate domains composed of either all open or all closed bonds, each containing a single microstate. At some point, the open-bond domain becomes contaminated by one or more closed bonds, causing a self-perpetuating movement toward inflation.

\section{Conclusions}

The current model provides a mechanistic approach to cosmic inflation in which the phase transitions that accompany the initiation and termination of the process are a natural consequence of the stochastic evolution of microstates that determine the allowable degree of entropy within the system. The properties of the network provide the framework for an orderly transition to post-inflationary cosmic expansion without imposing gravitational effects or specialized quantum activity (Ringeval, Suyama, \& Takahashi, 2010; Peebles \& Ratra, 2002). Despite the speculative nature of the proposed mechanism for termination of cosmic inflation, it may nevertheless act as a catalyst for further investigation of the potential role of entropy in this process.

\section{References}

Annett, J. (2004). Superconductivity, Superfluids and Condensates. New York, USA: Oxford University Press. 
Bousso, R., Harlow, D., \& Senatore, L. (2015). Inflation after false vacuum decay: Observational prospects after Planck. Phys. Rev. D., 91, 083527. https://doi.org/10.1103/PhysRevD.91.083527

Cantor, J. (2016). Is the Lorentz factor a probability function in superfluid spacetime?. Applied Physics Research, 8. https://doi.org/10.5539/apr.v8n3p1

Carroll, S. M., \& Chatwin-Davies, A. (2018). Cosmic equilibration: A holographic no-hair theorem from the generalized second law. Phys. Rev. D., 97, 046012. https://doi.org/10.1103/PhysRevD.97.046012

Carroll, S. M., \& Chen, J. (2004). Spontaneous inflation and the origin of the arrow of time. arXiv:hep-th/0410270.

De Gennes, P. G. (1976). On a relation between percolation theory and the elasticity of gels. J. Physique Lett., 37, $1-2$.

Easson, D. A., Frampton, P. H., \& Smoot, G. F. (2012). Entropic inflation. International Journal of Modern Physics A., 27, 1250066.

Einhorn, M. B., Stein, D. L., \& Toussaint, D. (1980). Are grand unified theories compatible with standard cosmology?. Phys. Rev. D., 21, 3295-3298.

Farago, O., \& Kantor, Y. (2000). Entropic elasticity of phantom percolation networks. Europhys. Lett., 52, 413-419.

Golden, K. M. (1997). Percolation models for porous media. In U. Hornung (Ed.), Homogenization and Porous Media (pp. 27-43). New York, NY: Springer.

Guth, A. H. (1981). Inflationary universe: A possible solution to the horizon and flatness problems. Phys. Rev. D., 23, 347-356.

Huang, K. (1995). Bose-Einstein condensation and superfluidity. In A. Griffin, D. W. Snoke, \& S. Stringar (Eds.), Bose-Einstein Condensation (pp. 31-50). Cambridge, England: Cambridge University Press.

Huang, K., Low, H.-B., \& Roh-Suan, T. (2012). Scalar field cosmology: I. Asymptotic freedom and the initial-value problem. Class. Quantum Grav., 29, 155014. https://doi.org/10.1088/0264-9381/29/15/155014

Jedamzik, K., Pogosian, L., \& Zhao, G. B. (2021). Why reducing the cosmic sound horizon alone can not fully resolve the Hubble tension. Commun. Phys., 4, 123. https://doi.org/10.1038/s42005-021-00628-x

Kapitulnik, A., Aharony, A., Deutscher, G., \& Stauffer, D. (1983). Self-similarity and correlations in percolation. J. Phys. A., 16, L269-L274. https://doi.org/10.1088/0305-4470/16/8/003

Liberati, S., \& Maccione, L. (2014). Astrophysical constraints on Planck scale dissipative phenomena. Phys. Rev. Lett., 112, 151301. https://doi.org/10.1103/PhysRevLett.112.151301

Linde, A. D. (1982). A new inflationary universe scenario: A possible solution of the horizon, flatness, homogeneity, isotropy and primordial monopole problems. Phys. Lett. B., 108, 389-393.

Linde, A. D. (1983). Chaotic inflation. Phys. Lett. B., 129, 177-181.

Papon, P., Leblond, J., \& Meijer, P. H. E. (2006). The Physics of Phase Transitions: Concepts and Applications (2nd ed.). Berlin, Germany: Springer.

Patel, V. M., \& Lineweaver, C. H. (2017). Solutions to the cosmic initial entropy problem without equilibrium initial conditions. Entropy, 19, 411. https://doi.org/10.33, 90/e19080411

Peebles, P. J. E., \& Ratra, B. (2002). The cosmological constant and dark energy. https://doi.org/10.1103/RevModPhys.75.559

Perlmutter, S., Aldering, G., Della Valle, M., Deustua, S., Ellis, R. S., Fabbro, A., et al.. (1998). Discovery of a supernova explosion at half the age of the Universe. Nature, 391, 51-54.

Redner, S. (2007). Fractal and multifractal scaling of electrical conduction in random resistor networks. Retrieved from https://arxiv.org/abs/0710.1105

Riess, A., Filippenko, A. V., Challis, P., Clocchiatti, A., Diercks, A., Garnavich, P. M., et al.. (1998). Observational evidence from supernovae for an accelerating universe and a cosmological constant. Astron. J., 116, 1009-1038.

Ringeval, C., Suyama, T., Takahashi, T., Yamaguchi, M., \& Yokoyama, S. (2010). Relation between inflation and dark energy. J. Phys.: Conf. Ser., 259, 012082. https://doi.org/10.1088/1742-6596/259/1/012082 
Spolyar, D. (2011). SuperCool inflation: A graceful exit from eternal inflation at LHC scales and below. arXiv:1111.3629 [astro-ph.CO].

Starobinsky, A. A. (1979). Spectrum of relict gravitational radiation and the early state of the universe. JETP Lett., 30, 682-685.

Tegmark, M. (2005). What does inflation really predict?. Journal of Cosmology and Astroparticle Physics, 1. https://doi.org/10.1088/1475-7516/2005/04/001

Uzan, J.-P. (2015). Inflation in the standard cosmological model. Comptes Rendus Physique, 16, 875-890.

Volovik, G. E. (2013). The superfluid universe. In K.-H. Bennemann, \& J. B. Ketterson (Eds.), Novel Superfluids (Vol. 1, pp. 570-618). Oxford, England: Oxford Univ. Press.

\section{Copyrights}

Copyright for this article is retained by the author(s), with first publication rights granted to the journal.

This is an open-access article distributed under the terms and conditions of the Creative Commons Attribution license (http://creativecommons.org/licenses/by/4.0/). 